\title{
Genome-wide analysis of palindrome formation
}

\author{
Scott J. Diede ${ }^{1}$, Hisashi Tanaka ${ }^{2}$, Donald A. Bergstrom ${ }^{3}$, Meng-Chao Yao ${ }^{4}$, and Stephen J. \\ Tapscott $^{1}$ \\ ${ }^{1}$ Divisions of Clinical Research and Human Biology, Fred Hutchinson Cancer Research Center, \\ Seattle, WA 98109 \\ 2 Department of Molecular Genetics and Lerner Research Institute, Cleveland Clinic, Cleveland, $\mathrm{OH}$ \\ 44195 \\ 3 Merck Research Labs, Merck \& Co, Inc., West Point, PA 19486 \\ ${ }^{4}$ Division of Basic Sciences, Fred Hutchinson Cancer Research Center, Seattle, WA 98109
}

\section{To the Editor}

In 2005, we reported a widespread and nonrandom distribution of DNA palindromes in cancer cells acting as a structural basis for subsequent gene amplification. These conclusions were based, in part, on the development of a new technique for the genome-wide analysis of palindrome formation (GAPF). This technique relies on the rapid intrastrand re-annealing of palindromic sequences under conditions that do not favor intermolecular annealing followed by S1 nuclease digestion of single-stranded DNA. Competitive hybridization of GAPF enriched DNA from control and cancer cells on an early generation spotted cDNA array resulted in cancer-specific GAPF-positive signals. Direct molecular analysis validated the fact that a subset of these GAPF-positive signals represented cancer-specific palindromes located at the boundary of gene amplicons ${ }^{1,2}$. However, in more recent studies using higher density tiling arrays that permit direct validation of a larger number of specific GAPF-positive signals, we have determined that a subset of GAPF-positive signals obtained using the original GAPF protocol are not associated with palindromes or other genome rearrangements. These GAPFpositive signals from non-palindromic regions correlate with regions of high CG content and high levels of $\mathrm{CpG}$ methylation ${ }^{3}$, both features known to increase the melting temperature of DNA. Therefore, the original GAPF technique enriches both for palindromes, through rapid intra-strand reannealing, and for regions of heavily methylated $\mathrm{CpG}$ islands that are selected because they do not fully denature at 100 degrees $\mathrm{C}$ in $100 \mathrm{mM} \mathrm{NaCl}$.

Increasing the stringency of the denaturation step in the GAPF assay by adding 50\% formamide eliminates the signals from DNA methylation; and the signal from palindromes can be selectively eliminated by cycling through two rounds of the original GAPF procedure (Fig. 1). This is because the intra-molecular palindromic hairpin has a variably sized non-annealed loop at the center of the palindrome that is cleaved by the initial nuclease S1 digestion. Following cleavage of the loop, the second denaturation results in separation of the complementary strands. Therefore, the original GAPF procedure can be modified to either enrich for palindromes or to enrich for methylated $\mathrm{CpG}$ rich regions. We have recently used a modified GAPF procedure to identify differentially methylated regions in human medulloblastomas ${ }^{3}$.

In conclusion, the GAPF-positive signals in our original study represented both palindromes and $\mathrm{CpG}$ island methylation. Our published ${ }^{1,2,3}$ and ongoing studies directly confirm that 
GAPF identifies palindromes associated with gene amplicons in cancers; however, the initial publication over-estimated the frequency of palindrome formation and the frequency of palindromes occurring in similar locations among different cancers.

\section{References}

1. Tanaka H, Bergstrom DA, Yao M-C, Tapscott SJ. Widespread and nonrandom distribution of DNA palindromes in cancer cells provides a structural platform for subsequent gene amplification. Nature Genetics 2005;37:320-7. [PubMed: 15711546]

2. Tanaka H, Cao Y, Bergstrom DA, Kooperberg C, Tapscott SJ, Yao M-C. Intrastrand annealing leads to the formation of a large DNA palindrome and determines the boundaries of genomic amplification in human cancer. Molec Cell Biol 2007;27:1993-2002. [PubMed: 17242211]

3. Diede SJ, Guenthoer J, Geng LN, Mahoney SE, Marotta M, Olson JM, Tanaka H, Tapscott SJ. Proc Natl Acad Sci USA. epub 2009.

4. Tanaka H, Bergstrom DA, Yao MC, Tapscott SJ. Nat Genet 2010;42:361.

5. Warburton PE, Giordano J, Cheung F, Gelfand Y, Benson G. Inverted repeat structure of the human genome: the X-chromosome contains a preponderance of large, highly homologous inverted repeats that contain testes genes. Genome Res 2004;14:1861-9. [PubMed: 15466286] 


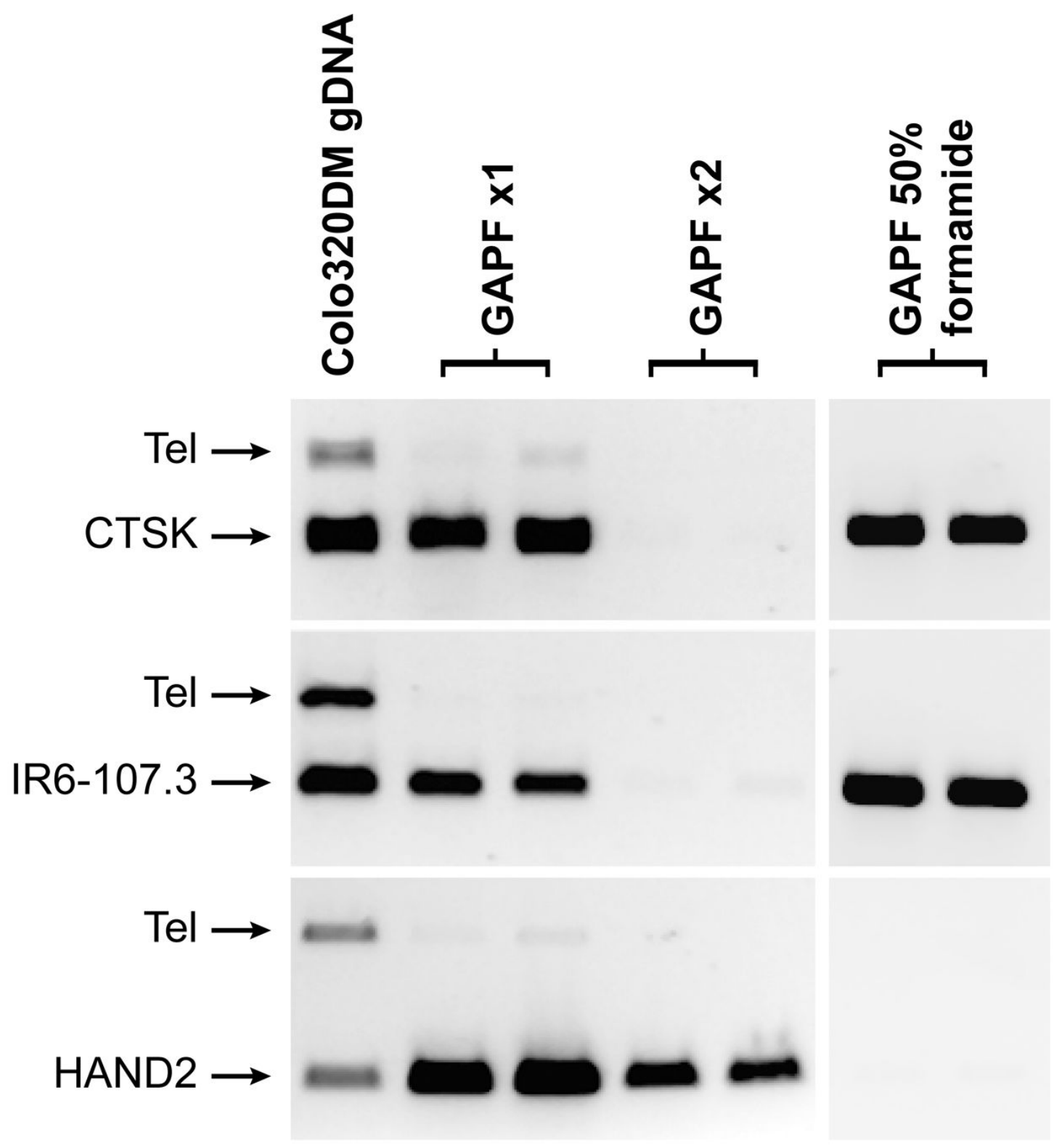

Figure 1.

Specific enrichment for either CpG methylation or DNA palindromes using the GAPF assay. Shown are results from Colo320DM cells of a PCR-based enrichment assay after one round of GAPF $($ GAPF $\times 1)$, two rounds of GAPF $($ GAPF $\times 2)$, or GAPF with $50 \%$ formamide added. The assay was performed in duplicate. PCR products obtained using unprocessed genomic DNA (gDNA) are included for comparison. As a negative control, the PCR product labeled as Tel amplifies a region on chromosome 1 that does not contain a DNA palindrome or CpG methylation, and primers to generate this fragment are added for multiplex PCR in each of the loci evaluated. A nonpalindromic GAPF-positive locus that is differentially CpG methylated $(H A N D 2)^{3}$ is recalcitrant to a second round of GAPF. The DNA palindrome at the CTSK locus is enriched after one round of GAPF but does not survive the second round. This pattern is also recapitulated for a naturally occurring DNA palindrome on chromosome 6 (IR6-107.3) ${ }^{5}$. The addition of $50 \%$ formamide during denaturation optimizes GAPF for DNA palindromes ${ }^{3}$, as 
shown by the enrichment for the CTSK and IR6-107.3 DNA palindromes, while abolishing the signal from the methylated HAND2 locus. 